\title{
Rad52 and Rad59 exhibit both overlapping and distinct functions
}

Feng, Qi; Düring, Louis; Antúnez de Mayolo, Adriana; Lettier, Gaëlle; Lisby, Michael; Erdeniz, Naz; Mortensen, Uffe Hasbro; Rothstein, Rodney

Published in:
DNA Repair

Link to article, DOI:

10.1016/j.dnarep.2006.08.007

Publication date:

2007

Document Version

Publisher's PDF, also known as Version of record

Link back to DTU Orbit

Citation (APA):

Feng, Q., Düring, L., Antúnez de Mayolo, A., Lettier, G., Lisby, M., Erdeniz, N., Mortensen, U. H., \& Rothstein, R. (2007). Rad52 and Rad59 exhibit both overlapping and distinct functions. DNA Repair, 6, 27-37.

https://doi.org/10.1016/j.dnarep.2006.08.007

\section{General rights}

Copyright and moral rights for the publications made accessible in the public portal are retained by the authors and/or other copyright owners and it is a condition of accessing publications that users recognise and abide by the legal requirements associated with these rights.

- Users may download and print one copy of any publication from the public portal for the purpose of private study or research.

- You may not further distribute the material or use it for any profit-making activity or commercial gain

- You may freely distribute the URL identifying the publication in the public portal 


\title{
Rad52 and Rad59 exhibit both overlapping and distinct functions
}

\author{
Qi Feng ${ }^{a}$, Louis Düring ${ }^{b}$, Adriana Antúnez de Mayolo ${ }^{a, 1}$, Gaëlle Lettier ${ }^{c}$, \\ Michael Lisby ${ }^{b}, \mathrm{Naz}_{\text {Erdeniz }}{ }^{d}$, Uffe H. Mortensen ${ }^{c}$, Rodney Rothstein ${ }^{a, *}$ \\ a Department of Genetics \& Development, Columbia University Medical Center, 701 West 168th Street, \\ New York, NY 10032-2704, USA \\ ${ }^{\mathrm{b}}$ Institute of Molecular Biology and Physiology, University of Copenhagen, Øster Farimagsgade 2A, \\ DK-1353 Copenhagen K, Denmark \\ c Center for Microbial Biotechnology, BioCentrum-DTU, Technical University of Denmark, Building 223, \\ DK-2800 Lyngby, Denmark \\ d Department of Molecular and Medical Genetics, Oregon Health Sciences University, \\ 3181 SW Sam Jackson Park Road, Mail Code L103, Portland, OR 97201, USA
}

\section{A R T I C L E I N F O}

\section{Article history:}

Received 7 July 2006

Received in revised form

9 August 2006

Accepted 9 August 2006

Published on line 20 September 2006

\section{Keywords:}

Rad52

Rad59

Recombination foci

Homologous recombination

Saccharomyces cerevisiae

\section{Abbreviations:}

YFP, yellow fluorescent protein; CFP, cyan fluorescent protein; DSB, DNA double-strand break; HR, homologous recombination; $\mathrm{dHJ}$, double Holliday junction; Hs, Homo sapiens; Sc, Saccharomyces cerevisiae; NLS, nuclear localization signal

\begin{abstract}
A B S T R A C T
Homologous recombination is an important pathway for the repair of DNA double-strand breaks (DSBs). In the yeast Saccharomyces cerevisiae, Rad52 is a central recombination protein, whereas its paralogue, Rad59, plays a more subtle role in homologous recombination. Both proteins can mediate annealing of complementary single-stranded DNA in vitro, but only Rad52 interacts with replication protein A and the Rad51 recombinase. We have studied the functional overlap between Rad52 and Rad59 in living cells using chimeras of the two proteins and site-directed mutagenesis. We find that Rad52 and Rad59 have both overlapping as well as separate functions in DSB repair. Importantly, the N-terminus of Rad52 possesses functions not supplied by Rad59, which may account for its central role in homologous recombination.
\end{abstract}

(c) 2006 Elsevier B.V. All rights reserved.

\footnotetext{
* Corresponding author. Tel.: +1 212305 1733; fax: +1 2129232090.

E-mail address: rothstein@cancercenter.columbia.edu (R. Rothstein).

1 Present address: Sylvester Comprehensive Cancer Center, University of Miami Medical Center, 1550 NW 10th Avenue, Miami, FL 33136 , USA.

1568-7864/\$ - see front matter (C) 2006 Elsevier B.V. All rights reserved.

doi:10.1016/j.dnarep.2006.08.007
} 
1.

\section{Introduction}

DNA double-strand break (DSB) repair is essential for protecting genomic integrity in all organisms. In the yeast Saccharomyces cerevisiae, the major pathway for DSB repair is homologous recombination (HR). HR is catalyzed by proteins encoded by the RAD52 epistasis group including RFA1-3, RAD50, MRE11, XRS2, SAE2, RAD51, RAD52, RAD54, RAD55, RAD57 and RAD59. Recombinational repair of DSBs can be explained by two different repair models, the canonical DNA double-strand repair model for homologous recombination that includes a double Holliday junction ( $\mathrm{dHJ}$ ) as a repair intermediate and the synthesis-dependent strand-annealing model that does not involve the formation of a $\mathrm{dHJ}$ (reviewed in [1]). A key step in the repair of most DNA DSBs by HR is the strand invasion reaction. In vitro, strand invasion can be efficiently performed by the collaborative effort of RPA, Rad51 and Rad52 according to the following scheme: the invasive single-stranded DNA (ssDNA) is bound by RPA to eliminate formation of inhibitory secondary DNA structures. However, RPA binding renders ssDNA inaccessible to Rad51, the recombinase that catalyzes the subsequent strand invasion reaction. Hence, RPA needs to be removed from the ssDNA prior to Rad51 binding. This step is mediated by Rad52, a protein that interacts with both RPA and Rad51 [2-7]. However, strand invasion in living cells is likely to be more complicated because of the participation of other protein factors of the RAD52 epistasis group. For example, yeast contains a Rad52 paralogue, Rad59, which appears to be a truncated version of Rad52 with homology only to the N-terminal region of Rad52. At present, the functions of Rad59 in DNA DSB repair and HR are poorly understood at the molecular level.

RAD59 was originally discovered on the basis of its role in RAD51-independent spontaneous mitotic recombination between inverted repeats [8]. Subsequently, two distinct pathways depending on either Rad59 or Rad51, but both requiring Rad52, have been defined for inverted repeat recombination, recombinational rescue of short telomeres and break-induced replication [8-10]. Homologues of Rad59 have been identified in a number of other organisms including lower eukaryotes, e.g. Kluyveromyces lactis and Eremothecium gossypii [11,12] and higher eukaryotes, e.g. mouse and human (Rad52B) [13], although it remains unclear whether these homologues are functional equivalents of the yeast protein.

The crystal structure of the N-terminal domain of the HsRad52 protein has been determined and reveals an undecameric ring $[14,15]$. The overall structure resembles a mushroom, consisting of a stem that contains highly conserved hydrophobic amino acid residues and a domed cap. By electron microscopy, it has been shown that full-length Rad52 forms heptameric ring structures [16]. Rad52 multimers likely exist in vivo since genetic studies have shown that $\mathrm{N}$ - and C-terminal rad52 mutations display intragenic complementation $[17,18]$. Rad59 has been suggested to form multimers similar to those formed by Rad52 or to form heteromeric rings with Rad52. This view is supported by the findings that Rad59 interacts with Rad52 as well as with itself [19]. Moreover, a sequence comparison of Rad52 and Rad59 reveals that the amino acid residues involved in Rad52 monomer-monomer interaction are the most highly conserved between the two proteins.

The largest difference between Rad52 and Rad59 is the C-terminal extension of Rad52 (amino acids 232-504), which is not conserved in Rad59. This region of Rad52 contains important functions including the Rad51 binding domain $[20,21]$, which is required for Rad52 to efficiently mediate Rad51 catalyzed strand exchange [3]. In agreement with this domain organization, it was found that overexpression of Rad51 suppresses the sensitivity to the alkylating agent methyl methanesulfonate of a rad52 mutant lacking the Cterminal domain [17]. In addition to a Rad51 binding domain, the C-terminus of human Rad52 has been shown to contain an RPA binding domain [22,23]. In this context, it is important to note that Rad52 is recruited to sites of DNA damage by RPA, whereas the recruitment of Rad51 and Rad59 is strictly dependent on Rad52 [24]. Both Rad52 and Rad59 accumulate into focal assemblies at DSBs. Co-immunoprecipitation experiments have identified both Rad51-Rad52-Rad59 and RPA-Rad52-Rad59 complexes, but no association of Rad59 with Rad51 or RPA is observed in the absence of Rad52 [19].

Genetic studies suggest that Rad52 and Rad59 have overlapping functions. Specifically, rad52-R70K and rad59 $\Delta$ strains display synergistic sensitivity to $\gamma$-irradiation and a synergistic defect in meiosis [25]. Moreover, overexpression of Rad52 partially suppresses the $\gamma$-ray sensitivity of rad59 $\Delta$ cells [8]. This view is supported by biochemical analyses, which show that both proteins bind DNA and stimulate DNA annealing [21,26-28]. Considering the absence of Rad51 and RPA binding domains in Rad59, it is not surprising that rad52 $\Delta$ strains display a much more severe phenotype than rad59 $\Delta$ strains and that overexpression of Rad59 cannot suppress a rad52 $\Delta$ phenotype [8]. Consistent with these observations, Rad52 but not Rad59 catalyzes the annealing of RPA-bound ssDNA in vitro [28]. However, other functional differences between Rad59 and Rad52 may exist. In this study, we have analyzed the functional overlap and differences between these two proteins in HR and DNA DSB repair. Hence, we find that the N-terminus of Rad52 contains functions that are unique to Rad52 and we identify a number of amino acid residues in the N-terminus of Rad52 that are involved in functions that overlap with those of Rad59. The latter amino acid residues are likely involved in a novel common function of Rad52 and Rad59 since none of the amino acid residues are predicted to be located in the putative DNA binding groove of Rad52 or at the interface between individual Rad52 subunits.

\section{Materials and methods}

\subsection{Media, strains and genetic methods}

Yeast extract-peptone-dextrose (YPD) medium, synthetic complete (SC) medium and SC lacking specific amino acids were prepared as described previously [29]. Standard yeast manipulations were used for mating, sporulation, dissection and replica plating [30]. Lithium acetate transformation was employed [31]. All strains are RAD5 derivatives of W303 (Supplementary Table). 
The procedure of alanine substitution of residues in the Rad52 N-terminus was described previously [32]. Mutants of RAD52 that display $\gamma$-ray sensitivity only in the rad59 $\Delta$ background are analyzed in this study.

Selected mutants of Rad52 were fused to yellow fluorescent protein (YFP) or cyan fluorescent protein (CFP) and analyzed by fluorescence microscopy as described previously [33].

\section{2. $\gamma$-Ray sensitivity}

Plasmid-borne rad52 mutants were transformed into a rad52 $\Delta$ rad59 $\Delta$ strain (W2081-1D). The transformants were tested for $\gamma$-ray sensitivity as described previously [32]. Mutations that lowered the survival by approximately 100 -fold or more after exposure to $200 \mathrm{~Gy}$ compared to a transformant harboring a wild-type RAD52 plasmid (pWJ1561) were designated class E mutants (for a description of class A through D mutants, see [32]) and selected for further analyses. Genomically integrated mutants were analyzed similarly except that cells were grown in YPD medium instead of SC-Trp. The $\mathrm{LD}_{37}$ was calculated as $-\ln (2.7) /$ slope, where the slope is determined from the linear fit to the $\ln (\%$ survival) versus $\gamma$-ray dose plot.

\subsection{Mitotic recombination rates and direct-repeat recombination rates}

Mitotic recombination between leu2- $\Delta$ BstEII and leu2- $\Delta$ EcoRI heteroalleles was measured in diploids as described previously [32] except that different media were used as indicated. The diploid strains, listed in Supplementary Table, were grown in SC medium before plating on SC-Leu plates.

The rate of leu2 direct-repeat recombination was determined in haploid strains as described in [32] except that cells were grown in SC medium before plated on SC-Leu plates.

\subsection{Construction of Rad59-Rad52 chimera plasmids}

Chimera A consists of Rad59 residues 1-175 fused to Rad52 residues 169-504. Chimera B consists of full-length Rad59 fused to Rad52 residues 232-504. In brief, the chimera constructs were made as follows. First, sequences were amplified by PCR from pRS416-Rad59 [37] as a template and primers A: 5'-GGCGAATGGATGTTATAGAT-3' and B: 5'CTTCTTTAACGCATCGCCTA-3' (fragment AB) or A: 5'-GGCGAATGGATGTTATAGAT- $3^{\prime}$ and C: 5'-GGCGAATGGATGTTATAGAT-3' (fragment AC). Second, Rad52 fragments were amplified by PCR using pWJ1561 as a template with primer E: 5'-TAGGCGATGCGTTAAAGAAGTCTTTGAGAGGGTTTGGTAA3' and D: 5'-CGCGAATTCCGGGCGCTAACCTGGACCTTCTAGAAGGCGGCCAGGAAGCGTTTCAAGTAGGC-3' (fragment ED) or F: 5'-CTAAAGGCACGCATATCAAAAATAAAAGAAGGCAATTGAC$3^{\prime}$ and D: 5'-CGCGAATTCCGGGCGCTAACCTGGACCTTCTAGAAGGCGGCCAGGAAGCGTTTCAAGTAGGC-3' (fragment FD). Subsequently, the partially overlapping fragments $A B$ and $E D$ or $A C$ and FD were fused in a second round of PCR using primers $A$ and $D$. The resulting fragments $A B E D$ and ACFD were then digested by XmaI and $\mathrm{XbaI}$ and ligated into XmaI and XbaI digested pRS416-Rad59 [37] vector separately. The newly formed plasmids were digested with MscI and
BamHI and each chimera Rad52-Rad59 containing fragment was isolated and ligated into the SpeI (end filled out by T4 polymerase)-BamHI fragments of pWJ1561 and pWJ1562, respectively, to create pRS413-chimera A (pWJ1191), pRS413chimera B (pWJ1193), pRS423-chimera A (pWJ1192), and pRS423-chimera B ( $p$ WJ1194). The expression plasmids were verified by sequencing.

To tag the chimeras with YFP, first a CEN-based plasmid pWJ1213 for expression of Rad52-YFP from its endogenous promoter was constructed by PCR-amplifying a RAD52-YFP fragment from strain W3749-14C [34] using primers Rad52fwd2010 (5'-CCTTTGTTACAGCTAAGGC) and Rad52-down (5'-AATGAACCTAAGGATTCCGC). This fragment was cotransformed into yeast strain W1588-4C [35] for gap-repair with an SphI cut pRS413-Rad52 plasmid [32]. The gap-repaired plasmid (pWJ1213) was rescued from yeast and sequenced to confirm the correct reconstitution of the RAD52-YFP expression cassette. Next, plasmids pWJ1191 to pWJ1194 were digested with PstI and the chimera-containing fragments were gel-purified. Likewise, the pWJ1213 plasmid was digested with NheI and a fragment containing the 3 '-end of RAD52, YFP and the RAD52 teminator sequence was gel-purified. The two gel-purified restriction fragments were co-transformed into W1588-4C for gap-repair using the $320 \mathrm{bp}$ and $1737 \mathrm{bp}$ of flanking sequence homology on either side of PstI site. The resulting plasmids (pWJ1241 to pWJ1244) containing chimera A and B fused to YFP were rescued by preparation of genomic DNA [36] and transformation into DH10B competent E. coli cells. The constructs were verified by restriction analysis and transformed into appropriate yeast strains for analysis by fluorescence microscopy (see text).

To test the functionality of the YFP fusion proteins, pWJ1191-pWJ1194, pWJ1241-pWJ1244, pRS413, pWJ1564 and pWJ1565 were transformed into a rad59 $\Delta$ strain (W3251-27D). Individual transformants were grown in SC-His medium and then spotted in 10-fold serial dilutions onto SC-His plates before exposure to $\gamma$-irradiation. All plasmids are listed in Supplementary Table.

\subsection{Complementation tests, fluorescence microscopy and protein blotting}

The chimera plasmids were transformed into W1588-4C (wildtype), W3251-27D (rad59::LEU2), W3537-34A (rad52-207A), W3744-10D (rad52-327 $)$ or W2014-5C (rad52::HIS5) and $\gamma$ ray sensitivity was tested as described previously [32]. Likewise, the plasmids containing the YFP-tagged chimeras were transformed into W2312-11D (RAD52-CFP), W3744-10D (rad52327 $\Delta$ ), W3537-34A (rad52-207 $\Delta$ ), W3777-17A (rad52::HIS5), W4970-10B (RAD52-RFP rad59::HIS3), W5867-2B (CFP-RAD51 rad52::HIS5) and W5843-2C (CFP-RAD51). W6640-3B (RAD52YFP CFP-RAD51) acts as a control. Plasmids expressing chimeric proteins (pWJ1191-pWJ1194) were also transformed into UM56-1B (Rad52-207ه-YFP) to examine localization. The transformants were then prepared for fluorescence microscopy as described [33]. DNA was visualized by staining live cells with $10 \mu \mathrm{g} / \mathrm{ml}$ DAPI for $30 \mathrm{~min}$.

The expression level of chimera proteins was determined by protein blot analysis using an anti-Rad52 antibody as described previously [21]. 


\section{Results}

\subsection{Construction of Rad59-Rad52 chimeras}

First, we addressed the question of whether Rad59 is functionally equivalent to the conserved N-terminus of Rad52 $[19,27,37]$. To test this possibility, we constructed two chimeras of Rad59 and Rad52 in which the conserved Rad59 N-terminus or full-length Rad59 is extended with the Rad51 and RPA interacting C-terminal domain of Rad52 (Fig. 1A). In both cases, the final protein fusion was approximately the size of the fulllength Rad52 protein. More specifically, chimera A consists of the Rad59 residues that are most homologous to Rad52 (1-175) fused at the site of homology to Rad52 (residues 169-504). Chimera B contains the entire Rad59 open reading frame fused to residues 232-504 of Rad52. If the chimeras are expressed and sort to the nucleus, and if the N-termini of Rad52 and Rad59 have identical and interchangeable functions, then each chimera is expected to behave like genuine Rad52 protein. On the other hand, if the N-termini of Rad52 and Rad59 have distinct functions, then, at the least, the chimeras should complement a rad59 $\Delta$ strain.

\subsection{Rad59-Rad52 chimeras are expressed and sort to the nucleus}

To test expression of the two chimeras, total cell extracts were prepared from the transformed strains and analyzed by protein blotting using an anti-Rad52 antibody (Fig. 1B). Both of the chimeras appear slightly larger than Rad52 as judged by their electrophoretic mobility (compare the chimeric proteins to the endogenous Rad52 band in the rad59 $\Delta$ strain in Fig. 1B). In the rad59 $\Delta$ strain, chimera $A$ is only detected when it is expressed from a multi-copy plasmid, however a significant degradation band is also seen. Higher levels of chimera B are also seen when it is expressed from a multi-copy plasmid. In a rad52 $\Delta$ strain, chimera $A$ is barely detected from either single or multi-copy plasmids and significant levels of chimera $B$ are detected only when it is expressed from a multi-copy plasmid. These observations suggest that wild-type Rad52 protein aids in stabilizing the chimeras in vivo.

We have recently shown that Rad59 requires Rad52 for efficient nuclear localization [24]. Moreover, we have also identified a nuclear localization signal (NLS) of the pat7 type spanning residues 231-237, which is essential for the sorting of Rad52 to the nucleus (I. Plate, U. Mortensen, in preparation) [38]. Chimera A contains this NLS, but in chimera B the NLS is right at the Rad59-Rad52 junction and was changed from being a pat7 type to a conventional NLS-type during the construction [39]. Therefore, we tested whether both chimeras are correctly sorted to the nucleus by tagging them with YFP to determine their localization. The YFP-tagged chimeras behave similarly to the untagged chimeras in their complemention of the $\gamma$-ray sensitivity of a rad59s strain (data not shown). In wild-type or rad59 $\Delta$ cells, ectopic expression of either chimera allows proper localization to the nucleus (Fig. $1 \mathrm{C}$ and data not shown). Consistent with the protein blot analysis, chimera $\mathrm{A}$ is barely detectable in rad52 $\Delta$ cells (Fig. 1D), while chimera B is readily detected in the nucleus (Fig. 1D).

\subsection{Rad59-Rad52 chimeras complement the $\gamma$-ray sensitivity of rad59 $\Delta$, but not of rad52 $\Delta$ strains}

To study the activity of the chimeras, we first investigated whether they provide Rad59 function. Single- or multi-copy plasmids expressing either of the chimeras were transformed into a rad59 $\Delta$ strain and the transformants tested for $\gamma$ ray sensitivity. Even the reduced chimera A protein levels expressed from a single-copy plasmid can partially rescue the $\gamma$-ray sensitivity of a rad59 $\Delta$ strain (Fig. 2A). In contrast, chimera $B$ expression from a single copy plasmid fully rescues the $\gamma$-ray sensitivity of a rad59 $\Delta$ strain (Fig. 2A). Furthermore, both chimeras fully complement rad59 $\Delta$ when expressed from multi-copy plasmids (Fig. 2B). Therefore, we conclude that the function of Rad59 in $\gamma$-ray repair is maintained fully in chimera $\mathrm{B}$, but partially in chimera A.

Next, the ability of the chimeras to complement the $\gamma$ ray sensitivity of a rad52 $\Delta$ strain was determined. Whether expressed from a single- or multi-copy plasmid, the chimeras completely fail to rescue the $\gamma$-ray sensitivity of a rad52 $\Delta$ strain (Fig. 2C and D). In light of the fact that expression of chimera $B$ from a multi-copy plasmid results in near wild-type protein levels (compared to endogenous Rad52), we conclude that Rad59 cannot substitute for the functions normally provided by the $\mathrm{N}$ terminus of Rad52.

\subsection{Rad59-Rad52 chimeras interact with Rad51 but are recruited inefficiently to sites of DNA damage}

Next, we investigated whether the Rad52 portion of the chimera can engage Rad51 in DNA repair. Since the Rad52 segment of the chimera contains the Rad51 interaction domain, we tested whether Rad51 binding was functional in the two chimeras. Two-hybrid analysis indicates that chimera B, unlike Rad59 on its own, is capable of interacting with Rad51 although not as strongly as Rad52 (data not shown) [19]. However, this experiment does not reveal whether the interaction of chimera B with Rad51 is productive in DNA repair. To investigate this possibility, we took advantage of the fact that the DNA damage sensitivity of rad52-207 $\Delta$ and rad52-327 $\Delta$ truncation mutants lacking the Rad51 interaction domain can be rescued by intragenic complementation with rad52-2, which has a missense mutation in the Rad52 N-terminus $[17,18]$. This result shows that functions of Rad52 present in the N- and Cterminus can collaborate even when present in two different molecules, likely because the two mutant species form heteromeric rings.

Inspired by this observation, we tested whether the defects of strains expressing the rad52 truncation mutants can be suppressed by co-expression of the Rad59-Rad52 chimeras. Hence, single- or multi-copy plasmids expressing the chimeras were transformed into rad52-207 $\Delta$ and rad52$327 \Delta$ strains. The resulting transformants were tested for $\gamma$-ray sensitivity. As seen in Fig. 3A, the chimeras partially complement rad52-327 $\Delta$, demonstrating that both can form a complex with rad52-327 $\Delta$ and bind Rad51 to reconstitute a proficient DSB repair machinery. In contrast, neither chimera complements rad52-207 $\Delta$ (Fig. 3B). The difference between the two rad52 truncation mutants may be explained by the Rad52$207 \Delta$ protein lacking nuclear localization, while Rad52-327 $\Delta$ 
(A)

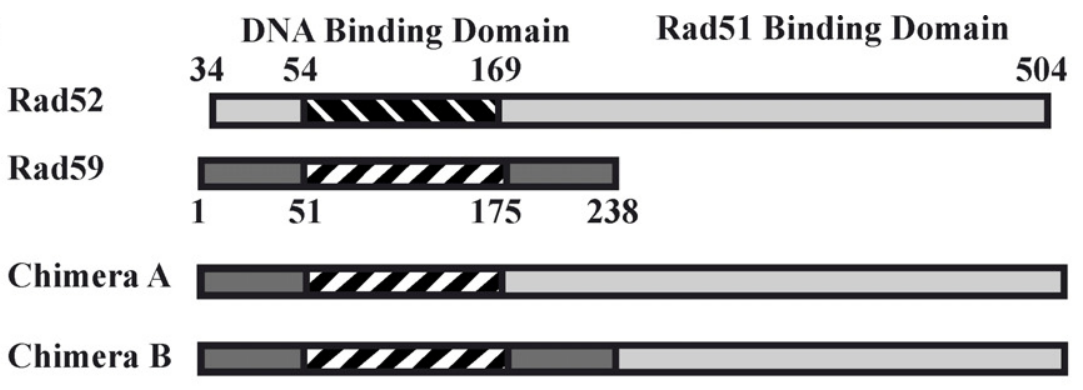

(B)

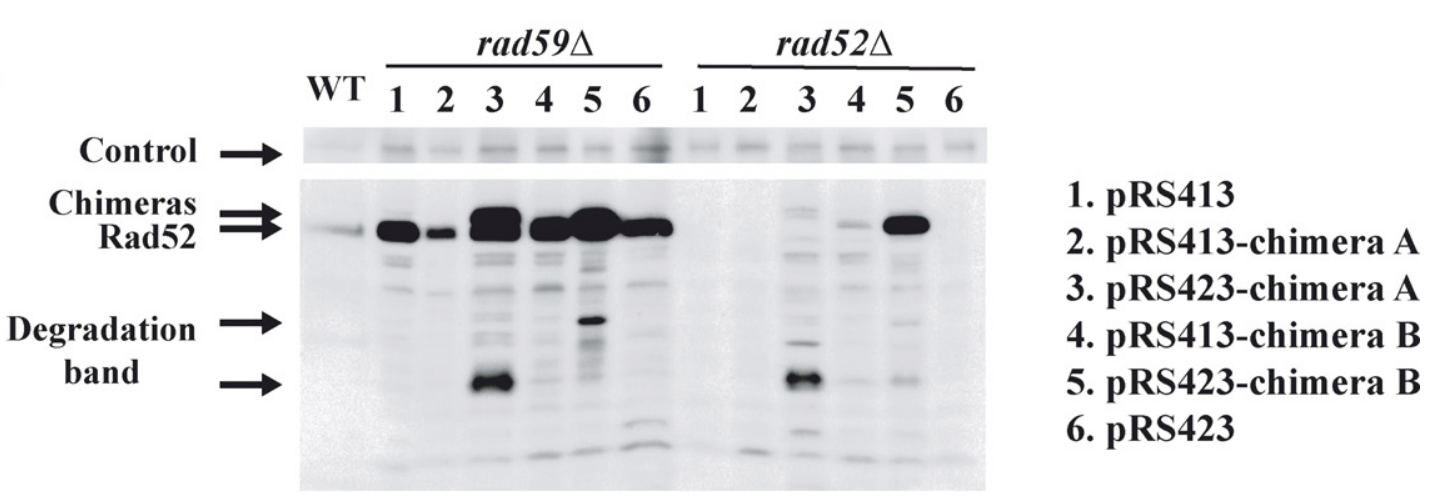

(C)
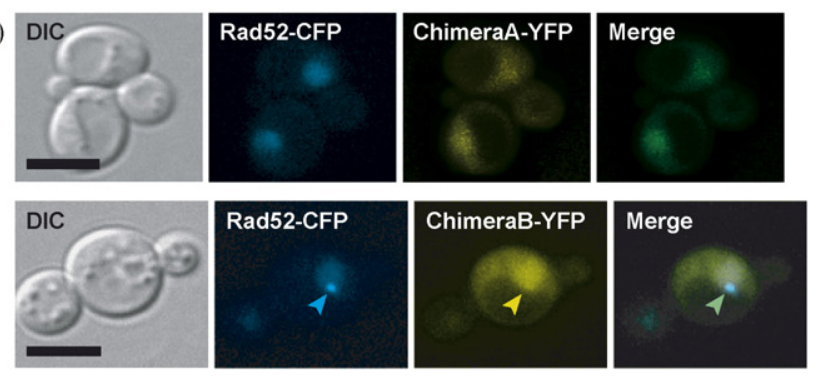

(D)
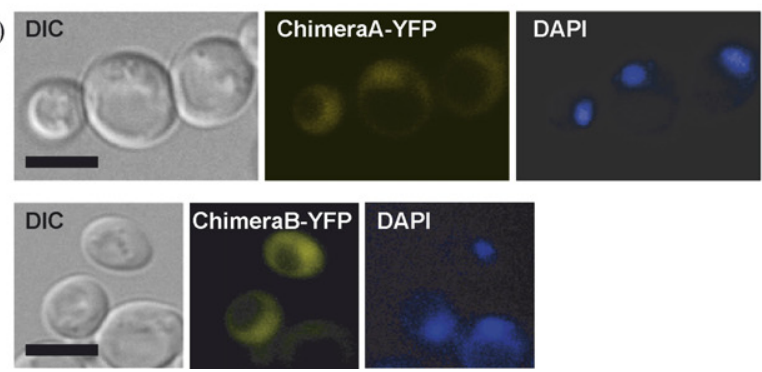

Fig. 1 - Characterization of Rad59-Rad52 chimeric proteins and cellular localization of chimeras. (A) Chimera constructs. Rad59 shares $32 \%$ sequence identity and $53 \%$ similarity with the $\mathrm{N}$-terminus of Rad52. Hatching indicates the highly conserved DNA binding domain and shading the less conserved amino acids. Chimera A consists of Rad59 residues 1-175 fused to Rad52 residues 169-504. Chimera B contains all of Rad59 fused to Rad52 residues 232-504. (B) Protein blot analysis of chimera proteins. Rad59-Rad52 chimeras expressed from single- or multi-copy vectors (pRS413 and pRS423, respectively) in rad59 $\Delta$ or rad52 $\Delta$ strains as indicated. A control cross-reacting protein is shown from an over-exposed film of the same gel. The endogenous wild-type Rad52 protein is visible both in WT and the rad59 $\Delta$ strains. (C) Localization of chimera A and B expressed from a multi-copy plasmid in RAD52 wild-type cells (W2312-11D). (D) Nuclear localization of chimeras in $\operatorname{rad52\Delta }$ (W3777-17A). Scale bar, $3 \mu \mathrm{m}$.

is proficient in nuclear recruitment (I. Plate, U. Mortensen, in preparation). Therefore, we tested the ability of the chimeras to support transport of Rad52-207 $\Delta$ into the nucleus. For microscopy analysis, the chimera plasmids were individually transformed into a strain expressing Rad52-207 $\Delta$-YFP (UM561B). Inspection of the transformants shows that Rad52-207 $\Delta$, which on its own resides in the cytoplasm, is transported into the nucleus by the chimeras (data not shown). These results 

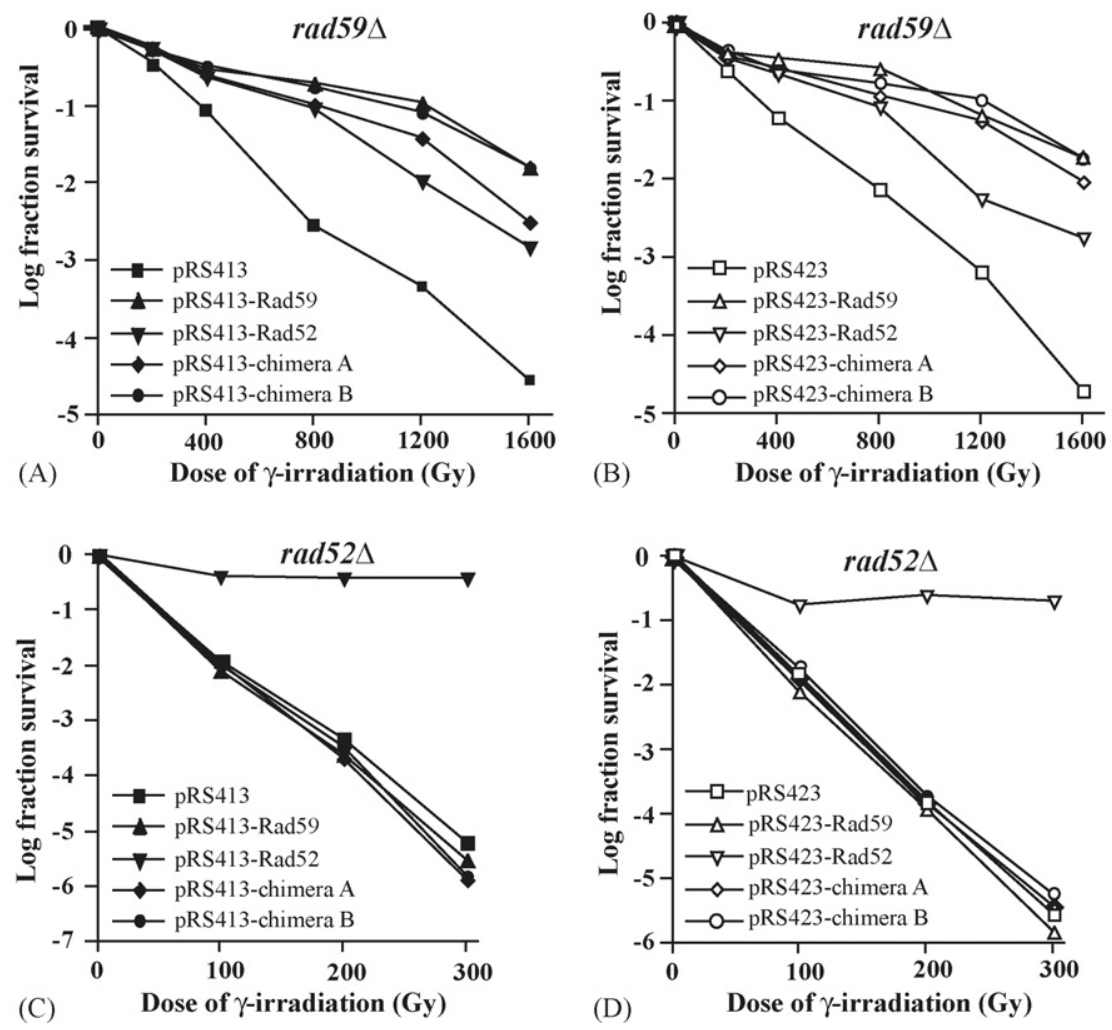

Fig. 2 - $\gamma$-Ray survival curves for Rad59-Rad52 chimeras expressed from single- or multi-copy vectors in rad59 $\Delta$ or rad52 $\Delta$ strains (W3251-27D and W2014-5C, respectively) as indicated. (A-D) $\square$, pRS413; $\Delta$, pRS413-Rad59; $\nabla$, pRS413-Rad52; pRS413-chimera-A; $\bullet$, pRS413-chimera-B; $\square$, pRS423; $\Delta$, pRS423-Rad59; $\nabla$, pRS423-Rad52; $\diamond$, pRS423-chimera-A; $\bigcirc$, pRS423-chimera-B.

indicate that the chimeras form a complex with the Rad52 truncations and are able to engage Rad51 in DNA repair. However, the chimeras lack functions other than those normally supplied by the Rad52 N-terminus.

Further, we tested whether the chimeras are recruited to sites of DNA repair by monitoring their localization relative to wild-type Rad52 tagged with cyan fluorescent protein (CFP) after exposing cells to $40 \mathrm{~Gy}$ of $\gamma$-irradiation. After this treatment, chimera B forms foci that co-localize with Rad52-CFP and CFP-Rad51 foci (data not shown). In rad52 $\Delta$ cells, chimera $B$ also forms foci after exposure to $\gamma$-irradiation, albeit less efficient (5\% of budding cells) compared to wild-type (90\%) (Fig. 3C and data not shown). All chimera B foci colocalized with CFPRad51 foci (Fig. 3C), further arguing that the repair defects of chimera $B$ in rad52 $\Delta$ cells are not due to a failure in binding Rad51. Taken together, we conclude that the chimera B does not form a fully functional "Rad52" protein due to the lack of functions normally supplied by the N-terminus of Rad52 and not provided by Rad59. The reduced efficiency of focus formation in response to $\gamma$-irradiation suggests that this function involves recruitment to the DNA lesion.

\subsection{Identification of novel rad52 mutants with a synthetic genetic interaction with rad59 $\Delta$}

To map the regions of Rad52 that have overlapping functions with Rad59, we screened a previously generated collection of plasmid-borne site-specific point mutations in the Rad52 $\mathrm{N}$-terminus for synthetic genetic interactions with rad59 $\Delta$ in an assay for $\gamma$-ray sensitivity (see Section 2) [32]. This strategy identified nine mutants, K61A, S68A, Y80F, R114A, Y182A, Y184A, K189A, Y192A, and K194A, which display synergistic sensitivity to $\gamma$-irradiation in a rad59 $\Delta$ background (Fig. 4). These mutants define a new class of rad52 mutants referred to as class E (for a definition of class A, B, C and $D$ mutations see [32]). The class E mutants were integrated into the genome for further characterization. First, survival curves were generated by exposing cells to various doses of $\gamma$-irradiation and scoring the percentage of surviving cells (Fig. 5 and data not shown). For the $\gamma$-ray survival curves, the corresponding $\mathrm{LD}_{37}$ was calculated as described in Section 2 (Table 1). Several of the identified mutants display stronger synthetic effects with rad59 $\Delta$ compared to those described for rad52-R70K, the original mutant defining this class of rad52 mutants [25]. The rad52-R114A single mutant is the most $\gamma$ ray resistant of the class $\mathrm{E}$ mutants with an $\mathrm{LD}_{37}$ indistinguishable from the wild-type. In contrast, the rad52-R114A rad59 $\Delta$ double mutant is highly sensitive to $\gamma$-irradiation although not as sensitive as the rad52 $\Delta$ rad59 $\Delta$ double mutant (Table 1). At the other end of the spectrum, rad52-Y182A is the most $\gamma$-ray sensitive single mutant $\left(\mathrm{LD}_{37}=82 \pm 0.5\right)$ and the rad52-Y182A rad59 double mutant displays $\gamma$-ray sensitivity similar to the rad52 $\Delta$ rad59 $\Delta$ double null. The $\mathrm{LD}_{37}$ of the remaining class $\mathrm{E}$ rad52 mutants fall in between these two 


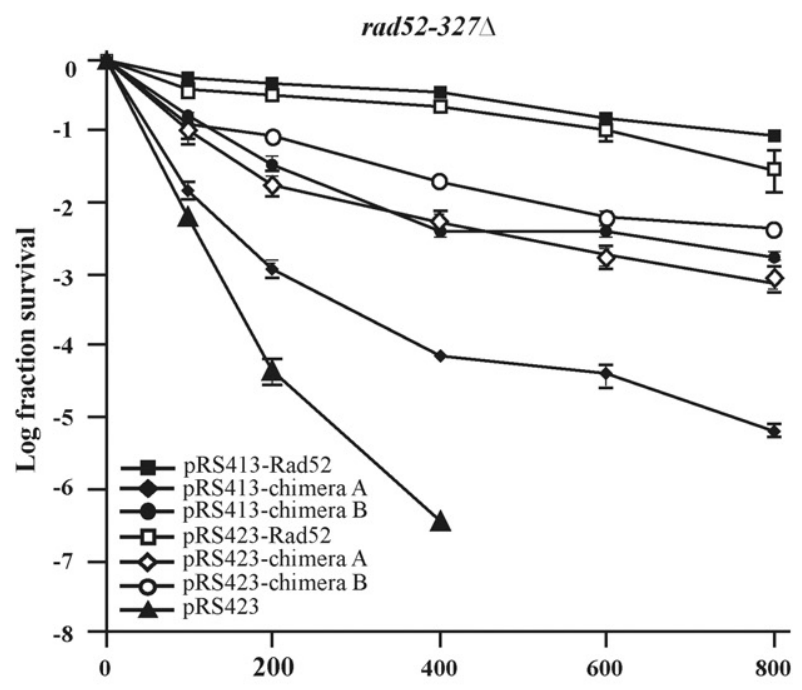

(A) Dose of $\gamma$-irradiation (Gy)

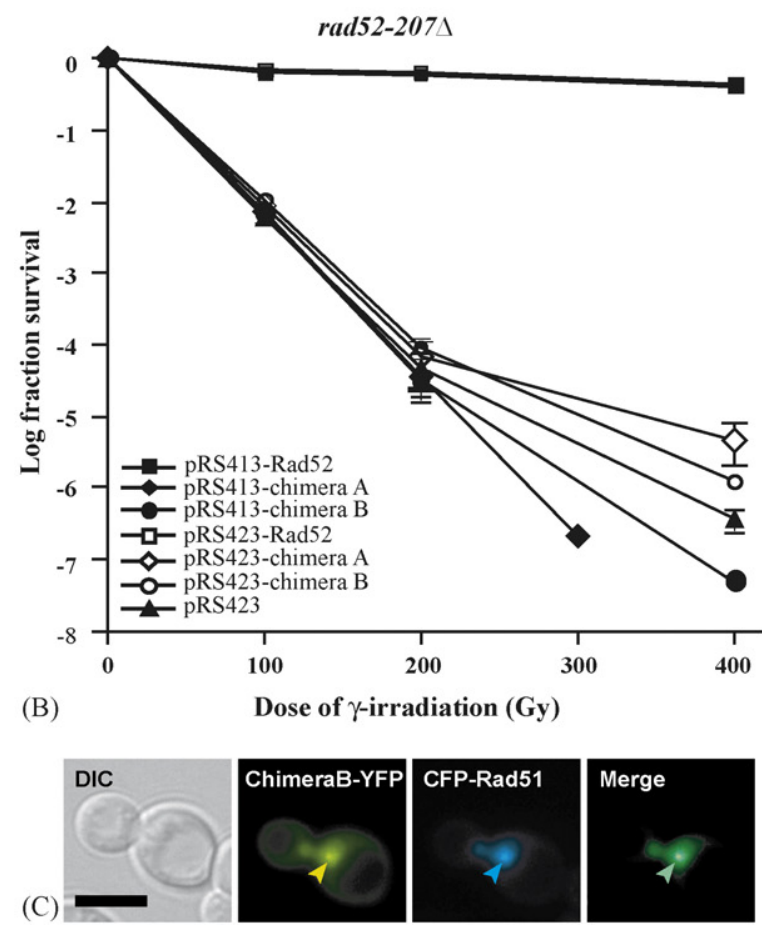

Fig. 3 - Complementation of the $\gamma$-ray sensitivity of $\operatorname{rad52-207\Delta ~(W3537-34A)~and~} \operatorname{rad52-327\Delta }$ (W3744-10D) by the chimeras. (A and B) $\square$, pRS413-Rad52; $\downarrow$, pRS413-chimera-A; $\bullet$, pRS413-chimera-B; $\square$, pRS423-Rad52; $\diamond$, pRS423-chimera-A; $\bigcirc$, pRS423-chimera-B; $\Delta$, pRS423. (C) Localization of $2 \mu \mathrm{m}$-expressed chimera B in CFP-RAD51 rad52 $\Delta$ (W5867-2B) $30 \mathrm{~min}$ after exposure to $40 \mathrm{~Gy}$ of $\gamma$-irradiation. Arrowheads mark co-localizing chimera B and Rad51 foci. Scale bar, $3 \mu \mathrm{m}$.

mutants. Of the class E mutants, rad52-K194A displays the largest difference in $\mathrm{LD}_{37}$ between the wild-type and rad59 $\Delta$ background (nine-fold). Thus, the ensemble of class $E$ amino acid residues are required for a function, which is shared by Rad59.
Next, the effect of the rad52 class E mutations on spontaneous heteroallelic and direct-repeat recombination was tested in both wild-type and rad59 $\Delta$ genetic backgrounds (Table 1). Surprisingly, no synthetic defect in recombination was observed when class $\mathrm{E}$ mutations were combined with rad59 $\Delta$. On the contrary, most of these double mutants displayed slightly elevated spontaneous recombination rates. In summary, in rad59 $\Delta$ strains, the rad52 class $E$ mutants fail to repair $\gamma$-ray induced damage even though they readily support mitotic homologous recombination. A similar separation of homologous recombination and repair of $\gamma$-rayinduced DNA damage was previously described for another class of rad52 mutants (class C) [32]. However, in contrast to rad52 class $\mathrm{E}$ mutants, the separation of function phenotype of class $C$ mutants can be observed even in the presence of Rad59.

Both wild-type and rad52 class $C$ mutant cells form spontaneous Rad52 foci in S phase in agreement with their ability to perform homologous recombination (Mortensen et al., PLoS Genetics, submitted for publication). To test whether Rad52 class E mutant protein behaves similarly, four class E mutants, rad52-S68A, K182A, K189A, and K194A, were tagged with YFP and their distribution in the cell visualized by fluorescence microscopy (Table 1). Similar to wild-type and Rad52 class $C$ mutants, the class E mutants form foci, indicating that the mutant protein is recruited to sites of DNA damage to engage in homologous recombination.

Overall, the rad52 class E mutants uncover regions in the Rad52 N-terminus that carry out essential functions in DNA double-strand break repair that can also be performed by Rad59. These functions are not essential for homologous interchromosomal heteroallelic recombination although this process may proceed slower in the absence of Rad59 as indicated by the large number of cells containing recombination foci in rad59 $\Delta$ strains.

\section{Discussion}

\subsection{Functional differences between the Rad52 and Rad59 N-termini}

In this study, we use Rad59-Rad52 chimeric proteins to demonstrate that the N-terminus of Rad52 provides unique function(s) to DSB repair that cannot be replaced by Rad59. In summary, the chimeras can complement the $\gamma$-ray sensitivity of a rad59 $\Delta$ strain but show no complementation of a rad52 $\Delta$ strain despite the fact that chimera $B$ is expressed, recruited to sites of DNA damage, and interacts with RPA, Rad52 and Rad51. Interestingly, both chimeras can complement the $\gamma$-ray sensitivity of rad52-327 $\Delta$, but not that of rad52-207 $\Delta$. Although Rad52-207 $\Delta$ does not contain a functional NLS, and does not sort to the nucleus on its own (I. Plate, U. Mortensen, in preparation), Rad52-207 $\Delta$-YFP is localized to the nucleus in the presence of both chimeras. Thus the lack of complementation between rad52-207 $\Delta$ and the chimeras cannot be explained by a failure of Rad52-207 $\Delta$ to localize to the nucleus indicating that the chimeras form complexes with the truncated Rad52 species. Furthermore, the $\gamma$-ray sensitivity of strains expressing the chimera-Rad52-207 $\Delta$ complexes, suggests 


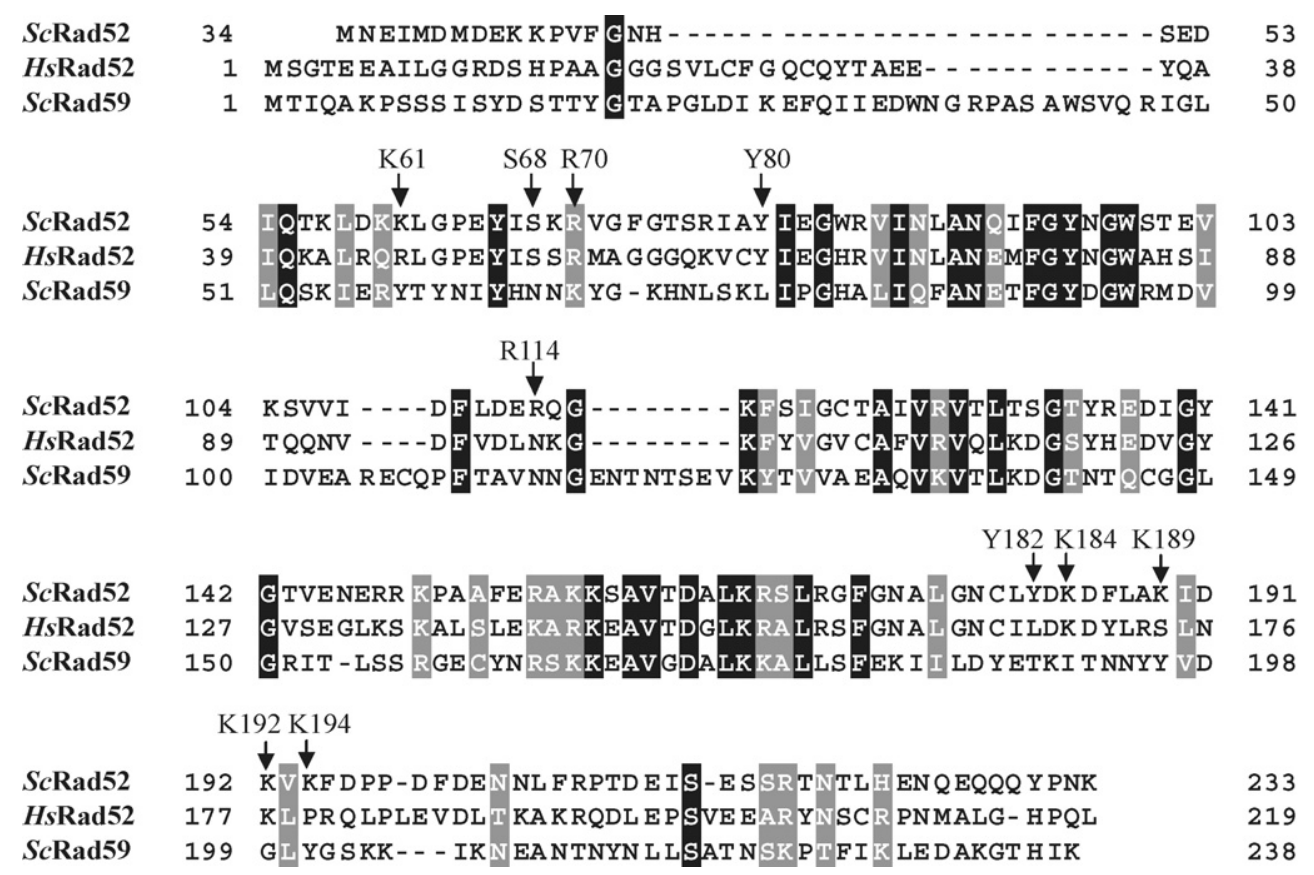

Fig. 4 - Alignment of Rad52 and Rad59 amino acid sequences. Identity indicated by black shading and similarity by grey shading. Alanine substituted residues with a synthetic genetic interaction with rad59 $\Delta$ are marked by arrowheads.

that the truncated Rad52-207 $\Delta$ protein does not benefit from the functional domains present in Rad52 amino acid residues 232-504 when they are recruited via the Rad59 portion of the chimera. We also observe that chimera B is inefficient at forming repair foci after exposure to $\gamma$-irradiation. This is consistent with the DNA repair defect of the chimeras and further indicates that functions normally supplied by the $\mathrm{N}$-terminus of Rad52 are not provided by Rad59 to ensure efficient recruitment of the chimeras to $\gamma$-ray-induced DNA damage.

\subsection{Novel rad52 mutants}

In addition to differences between Rad52 and Rad59, we identify nine mutations in RAD52, collectively referred to as class $E$, which confer synergistic $\gamma$-ray sensitivity with a rad59 $\Delta$ deletion, indicating that these residues encode functions common to the two proteins. The mutated residues lie within the highly conserved N-terminus of Rad52, but are not particularly conserved themselves. The low degree of evolutionary conservation among the mutated residues is consistent with

Table 1 - Effect of rad52 and rad52 rad59 $\Delta$ mutants on $\gamma$-ray sensitivity and mitotic heteroallelic and direct-repeat recombination

\begin{tabular}{|c|c|c|c|c|c|c|c|c|}
\hline \multirow[t]{2}{*}{ Allele } & \multicolumn{2}{|c|}{$\gamma$-Ray sensitivity $\left(\mathrm{LD}_{37}\right)^{\mathrm{a}}$} & \multicolumn{2}{|c|}{$\begin{array}{l}\text { Heteroallelic } \\
\text { recombination rate } \\
\left(\times 10^{-8}\right)^{\mathrm{b}}\end{array}$} & \multicolumn{2}{|c|}{$\begin{array}{l}\text { Direct-repeat } \\
\text { recombination } \\
\qquad\left(\times 10^{-6}\right)^{b}\end{array}$} & \multicolumn{2}{|c|}{$\begin{array}{l}\text { Spontaneous Rad52 } \\
\text { focus }^{d}(\% \text { cells) } \\
(G 1) /(S / G 2 / M)\end{array}$} \\
\hline & RAD59 & rad59 $\Delta$ & RAD59 & rad59 $\Delta$ & RAD59 & $\operatorname{Rad} 59 \Delta$ & RAD59 & rad59 $\Delta$ \\
\hline RAD52 & $392 \pm 74$ & $173 \pm 7$ & $102 \pm 15$ & $214 \pm 27$ & $45.3 \pm 11.6$ & $25.2 \pm 6.9$ & $0 / 14$ & $4.7 / 39.3$ \\
\hline $\operatorname{rad} 52 \Delta$ & $19 \pm 0.1$ & $21 \pm 1$ & $0.6 \pm 0.3$ & $1.8 \pm 0.5$ & $1.5 \pm 0.4$ & $2.5 \pm 0.7$ & 0 & 0 \\
\hline $\mathrm{K} 61 \mathrm{~A}$ & $105 \pm 4$ & $30 \pm 1$ & $\mathrm{ND}^{\mathrm{c}}$ & ND & $33.7 \pm 9.6$ & $42.2 \pm 10.4$ & ND & ND \\
\hline S68A & $116 \pm 14$ & $24 \pm 1$ & $127 \pm 22$ & $180 \pm 26$ & $46.6 \pm 11.4$ & $24.4 \pm 8.9$ & $0.7 / 24.1$ & $1.2 / 39.8$ \\
\hline Y80F & $156 \pm 17$ & $26 \pm 1$ & ND & ND & $26.4 \pm 9.0$ & $18.3 \pm 6.1$ & ND & ND \\
\hline R114A & $528 \pm 73$ & $67 \pm 4$ & $140 \pm 23$ & $400 \pm 47$ & $78.8 \pm 25.3$ & $38.5 \pm 13.2$ & $\mathrm{ND}$ & ND \\
\hline Y182A & $82 \pm 5$ & $33 \pm 3$ & ND & ND & $96.6 \pm 30.7$ & $49.8 \pm 17.0$ & $0.2 / 34.2$ & $0.7 / 39.2$ \\
\hline Y184A & $137 \pm 8$ & $28 \pm 2$ & $212 \pm 35$ & $400 \pm 52$ & $53.3 \pm 16.6$ & $40.5 \pm 16.6$ & ND & ND \\
\hline K189A & $260 \pm 53$ & $42 \pm 3$ & ND & ND & $54.3 \pm 17.5$ & $36.9 \pm 12.9$ & $1.6 / 9.8$ & $0.4 / 33.3$ \\
\hline Y192A & $198 \pm 15$ & $28 \pm 3$ & ND & ND & $65.8 \pm 16.0$ & $21.9 \pm 7.2$ & ND & ND \\
\hline K194A & $325 \pm 50$ & $37 \pm 3$ & $206 \pm 32$ & $440 \pm 59$ & $51.0 \pm 12.5$ & $46.1 \pm 14.1$ & $0.6 / 14.6$ & $0.7 / 58.7$ \\
\hline $\begin{array}{l}\text { a } L_{37} \mathrm{i} \\
\text { b Recon } \\
\text { c Not d } \\
\text { d The } p\end{array}$ & $\begin{array}{l}\text { Gy calculat } \\
\text { ination rat } \\
\text { ermined. } \\
\text { centage of }\end{array}$ & $\begin{array}{l}\text { described in Se } \\
\text { esented as eve }\end{array}$ & 2. & tion a & $\begin{array}{l}\text { previously } \\
\text { s(i). }\end{array}$ & & & \\
\hline
\end{tabular}




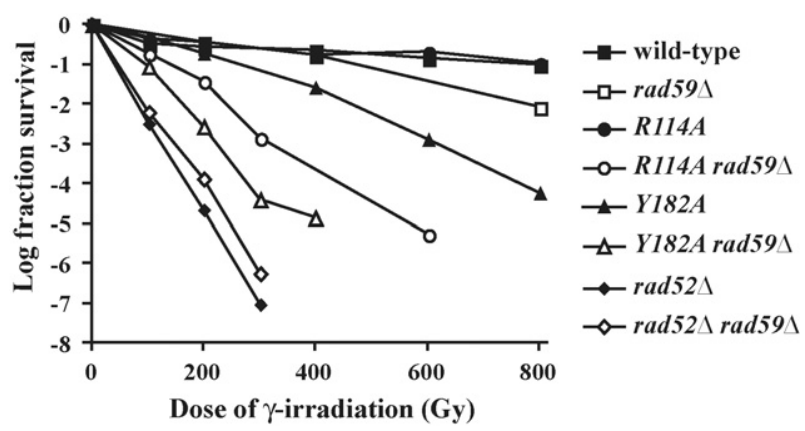

Fig. 5 - Synergistic $\gamma$-ray sensitivity between rad59 $\Delta$ and rad52 class E mutants. $\square$, wild-type (W1588-4C); $\square$, rad59 $\Delta$ (W3251-27D); ๑, rad52-R114A (J767); O, rad52-R114A rad59 $\Delta$ (W5394-7D); $\Delta, \operatorname{rad52-Y182A~(J75);~} \Delta, \operatorname{rad52-Y182A~rad59\Delta }$ (W5377-1A); $\diamond, \operatorname{rad52\Delta }$ (W2014-5C); $\diamond, \operatorname{rad52\Delta } \operatorname{rad59\Delta }$ (W2081-1D).

their mild phenotype in an otherwise wild-type genetic background. The severity of the mutations in a rad59 $\Delta$ background suggests that the defects conferred by the mutations can be complemented by Rad59 function.

In human Rad52, the amino acid residue that corresponds to R70 in S. cerevisiae, is involved in binding of single-stranded DNA and when mutated to alanine, DNA binding is severely reduced $[14,40]$. In $S$. cerevisiae, a rad52-R70A strain qualifies as a class $C$ mutant and is therefore very $\gamma$-ray sensitive even in the presence of Rad59. Hence, strongly compromised DNA binding in Rad52 is unlikely to be rescued by Rad59. In a rad52R7OK mutant, which is the founder of the class E mutants [25], an arginine residue is mutated to the chemically similar lysine residue. Likely, DNA binding is less affected in Rad52-R70K compared to Rad52-R70A where the positively charged arginine is changed to a small and neutral alanine residue. This difference may explain why DNA repair is compromised in the R70K protein only in the absence of Rad59. We therefore speculate that Rad59 rescues the defect of Rad52-R70K by stabilizing a weakened interaction with the DNA lesion. In this context it is interesting to note that by studying the dependency of direct repeat recombination on Rad59, Sugawara and colleagues also suggested that Rad59 acts by stabilizing recombination intermediates [41]. Possibly, this type of stabilization could be mediated via strand-annealing, which is a common activity of Rad52 and Rad59. Moreover, stabilization of Rad52 binding to a recombination substrate by Rad59 may be mediated via the ability of Rad59 to interact directly with Rad52, perhaps even by being fully integrated in a ring structure otherwise composed of Rad52 subunits $[37,42]$.

The human amino acid residues corresponding to the class E mutations discovered in this study map to the surface of the undecameric ring or at the base of the DNA binding groove in the structure of Rad52 [14] (Fig. 6). The class E mutations are therefore more likely to affect interaction with other proteins or with DNA rather than monomer-monomer interaction. Interestingly, two of the class E mutations (Y80F and K184A) severely reduce single-stranded DNA binding of human Rad52 $[14,40]$ although neither appears to be in direct contact with single-stranded DNA in the predicted structure [15]. This suggests that a wider range of residues in Rad52 contributes to efficient DNA binding. This interpretation is corroborated by the class $C$ mutations, which all map to the putative DNA binding groove in the human structure of Rad52 and have a
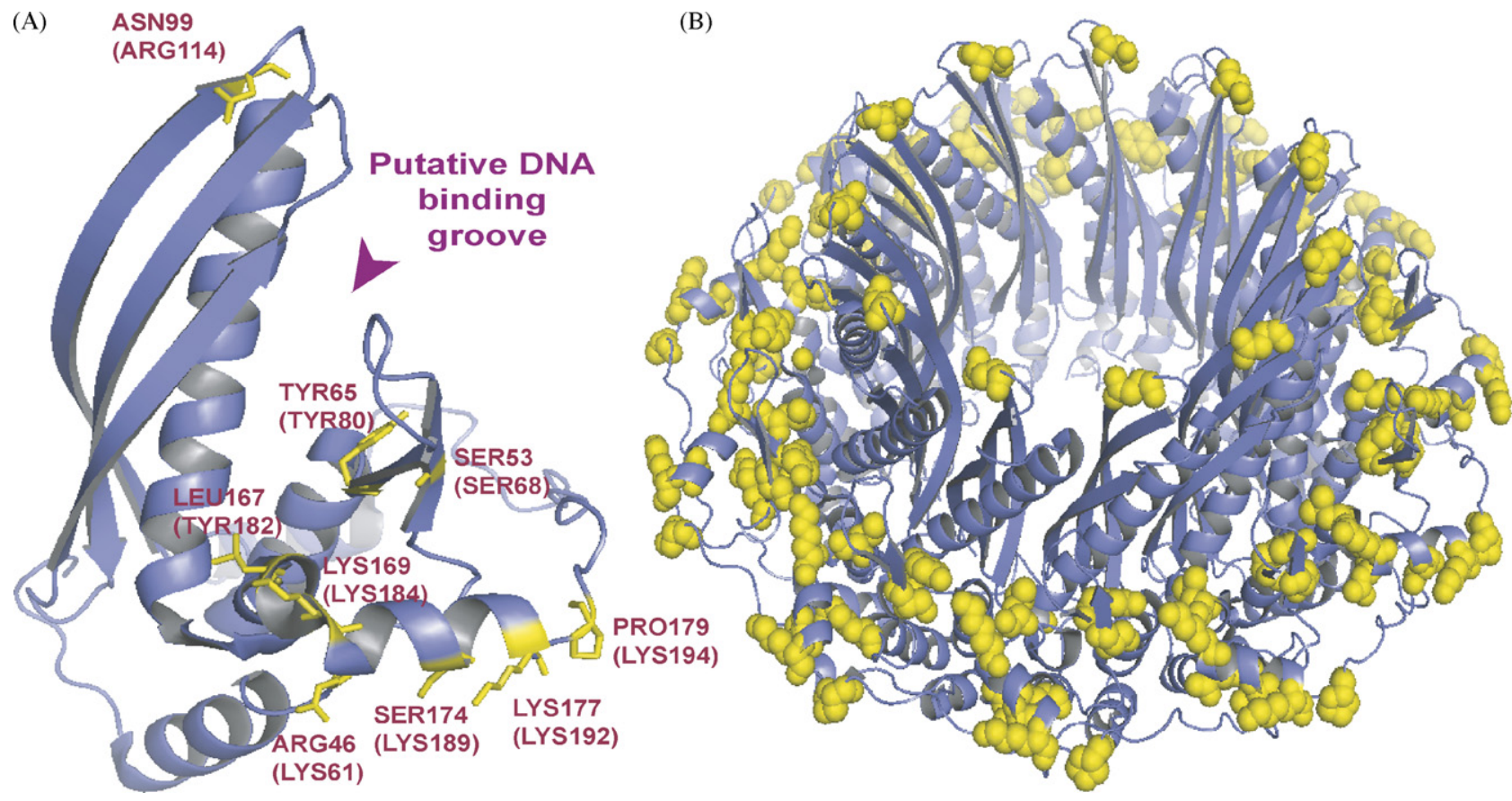

Fig. 6 - Modeling of rad52 class E mutations into the structure of human Rad52 (protein data bank ID 1KN0). The figures were created using Pymol software. (A) HsRad52 monomer. Labels indicate human amino acid residues and the corresponding S. cerevisiae residues in parentheses. (B) HsRad52 heptameric ring. Mutant residues showing a synergistic defect with rad59 $\Delta$ are shown in yellow. 
separation of function phenotype similar to that of the class $E$ mutants in a rad59 $\Delta$ background [32].

\subsection{Concluding remarks}

Based on the sum of evidence, we propose that binding of Rad52 to a DNA lesion is a multi-step process, where Rad52 is first recruited to the DNA lesion by its RPA interaction even in the absence of a functional DNA binding domain in the $\mathrm{N}$-terminus [24]. In agreement with this view, we have previously observed that Rad52-C180A, a class C mutant, can be recruited to a DNA double-strand break but fail to complete repair (Mortensen et al., PLoS Genetics, 2006 submitted for publication). All class E mutants spontaneously form repair foci during the cell cycle indicating that they are recruited to sites of DNA damage. After recruitment by RPA, efficient DNA binding/annealing by Rad52 requires both a functional DNA binding groove (defective in class $C$ mutants), which cannot be substituted by Rad59, and a second function (defective in class E mutants), which can be complemented by Rad59. This latter function could be a conformational change or an initial interaction with DNA guiding it to the DNA binding groove or stabilizing the bound DNA.

\section{Acknowledgments}

We thank members of the Rothstein laboratory and Lorraine Symington for helpful discussions concerning this work. This work was supported by NIH grant GM50237 (RR) and grants from the Tonnesen Foundation (RR), The Danish Natural Science Research Council (ML), the Alfred Benzon Foundation (ML), the Danish Medical Research Council and The Danish Technical Research Council (UHM), the Danish Biotech Research Academy, FOBI and the Technical University of Denmark for a PhD grant to GL.

\section{Appendix A. Supplementary data}

Supplementary data associated with this article can be found, in the online version, at doi:10.1016/j.dnarep.2006.08.007.

\section{REFERENCES}

[1] F. Paques, J.E. Haber, Multiple pathways of recombination induced by double-strand breaks in Saccharomyces cerevisiae, Microbiol. Mol. Biol. Rev. 63 (1999) 349-404.

[2] P. Sung, Function of yeast Rad52 protein as a mediator between replication protein A and the Rad51 recombinase, J. Biol. Chem. 272 (1997) 28194-28197.

[3] L. Krejci, B. Song, W. Bussen, R. Rothstein, U.H. Mortensen, P. Sung, Interaction with Rad51 is indispensable for recombination mediator function of Rad52, J. Biol. Chem. 277 (2002) 40132-40141.

[4] B. Song, P. Sung, Functional interactions among yeast Rad51 recombinase, Rad52 mediator, and replication protein $A$ in DNA strand exchange, J. Biol. Chem. 275 (2000) 15895-15904.

[5] J.H. New, T. Sugiyama, E. Zaitseva, S.C. Kowalczykowski, Rad52 protein stimulates DNA strand exchange by Rad51 and replication protein A, Nature 391 (1998) 407-410.
[6] A. Shinohara, T. Ogawa, Stimulation by Rad52 of yeast Rad51-mediated recombination, Nature 391 (1998) 404-407.

[7] F.E. Benson, P. Baumann, S.C. West, Synergistic actions of Rad51 and Rad52 in recombination and DNA repair, Nature 391 (1998) 401-404.

[8] Y. Bai, L.S. Symington, A Rad52 homolog is required for RAD51-independent mitotic recombination in Saccharomyces cerevisiae, Genes Dev. 10 (1996) 2025-2037.

[9] Q. Chen, A. Ijpma, C.W. Greider, Two survivor pathways that allow growth in the absence of telomerase are generated by distinct telomere recombination events, Mol. Cell. Biol. 21 (2001) 1819-1827.

[10] M.B. Vaze, A. Pellicioli, S.E. Lee, G. Ira, G. Liberi, A. Arbel-Eden, et al., Recovery from checkpoint-mediated arrest after repair of a double-strand break requires Srs2 helicase, Mol. Cell. 10 (2002) 373-385.

[11] F.S. Dietrich, S. Voegeli, S. Brachat, A. Lerch, K. Gates, S. Steiner, et al., The Ashbya gossypii genome as a tool for mapping the ancient Saccharomyces cerevisiae genome, Science 304 (2004) 304-307.

[12] M. van den Bosch, J.B. Zonneveld, P.H. Lohman, A. Pastink, Isolation and characterization of the RAD59 homologue of Kluyveromyces lactis, Curr. Genet. 39 (2001) 305-310.

[13] S. Hamimes, H. Arakawa, A.Z. Stasiak, A.M. Kierzek, S. Hirano, Y.G. Yang, et al., RDM1, a novel RNA recognition motif (RRM)-containing protein involved in the cell response to cisplatin in vertebrates, J. Biol. Chem. 280 (2005) 9225-9235.

[14] W. Kagawa, H. Kurumizaka, R. Ishitani, S. Fukai, O. Nureki, T. Shibata, et al., Crystal structure of the homologous-pairing domain from the human Rad52 recombinase in the undecameric form, Mol. Cell. 10 (2002) 359-371.

[15] M.R. Singleton, L.M. Wentzell, Y. Liu, S.C. West, D.B. Wigley, W. Kagawa, et al., Structure of the single-strand annealing domain of human RAD52 protein crystal structure of the homologous-pairing domain from the human Rad52 recombinase in the undecameric form, Proc. Natl. Acad. Sci. U.S.A. 99 (2002) 13492-13497.

[16] A.Z. Stasiak, E. Larquet, A. Stasiak, S. Muller, A. Engel, E. Van Dyck, et al., The human Rad52 protein exists as a heptameric ring, Curr. Biol. 10 (2000) 337-340.

[17] E.N. Asleson, R.J. Okagaki, D.M. Livingston, A core activity associated with the $\mathrm{N}$ terminus of the yeast RAD52 protein is revealed by $R A D 51$ overexpression suppression of C-terminal rad52 truncation alleles, Genetics 153 (1999) 681-692.

[18] K.L. Boundy-Mills, D.M. Livingston, A Saccharomyces cerevisiae RAD52 allele expressing a C-terminal truncation protein: activities and intragenic complementation of missense mutations, Genetics 133 (1993) 33-49.

[19] A.P. Davis, L.S. Symington, The Rad52-Rad59 complex interacts with Rad51 and replication protein A, DNA Repair (Amst) 2 (2003) 1127-1134.

[20] G.T. Milne, D.T. Weaver, Dominant negative alleles of RAD52 reveal a DNA repair/recombination complex including Rad51 and Rad52, Genes Dev. 7 (1993) 1755-1765.

[21] U.H. Mortensen, C. Bendixen, I. Sunjevaric, R. Rothstein, DNA strand annealing is promoted by the yeast Rad52 protein, Proc. Natl. Acad. Sci. U.S.A. 93 (1996) 10729-10734.

[22] L. Krejci, S. Van Komen, Y. Li, J. Villemain, M.S. Reddy, H. Klein, et al., DNA helicase Srs2 disrupts the Rad51 presynaptic filament, Nature 423 (2003) 305-309.

[23] M.S. Park, D.L. Ludwig, E. Stigger, S.H. Lee, Physical interaction between human RAD52 and RPA is required for homologous recombination in mammalian cells, J. Biol. Chem. 271 (1996) 18996-19000.

[24] M. Lisby, J.H. Barlow, R.C. Burgess, R. Rothstein, Choreography of the DNA damage response; spatiotemporal relationships among checkpoint and repair proteins, Cell 118 (2004) 699-713. 
[25] Y. Bai, A.P. Davis, L.S. Symington, A novel allele of RAD52 that causes severe DNA repair and recombination deficiencies only in the absence of RAD51 or RAD59, Genetics 153 (1999) 1117-1130.

[26] T. Sugiyama, J.H. New, S.C. Kowalczykowski, DNA annealing by RAD52 protein is stimulated by specific interaction with the complex of replication protein A and single-stranded DNA, Proc. Natl. Acad. Sci. U.S.A. 95 (1998) 60496054.

[27] G. Petukhova, S.A. Stratton, P. Sung, Single strand DNA binding and annealing activities in the yeast recombination factor Rad59, J. Biol. Chem. 274 (1999) 33839-33842.

[28] Y. Wu, T. Sugiyama, S.C. Kowalczykowski, DNA annealing mediated by Rad52 and Rad59 proteins, J. Biol. Chem. 281 (2006) 15441-15449.

[29] F. Sherman, Getting Started with Yeast, vol. 194, Academic Press, Inc., San Diego, 1991.

[30] F. Sherman, G.R. Fink, J.B. Hicks, Methods in Yeast Genetics, Cold Spring Harbor Laboratory, Cold Spring Harbor, NY, 1986.

[31] H. Ito, K. Fukada, A. Murata, Kimura, Transformation of intact yeast cells treated with alkali cations, J. Bacteriol. 153 (1983) 163-168.

[32] U.H. Mortensen, N. Erdeniz, Q. Feng, R. Rothstein, A molecular genetic dissection of the evolutionarily conserved $\mathrm{N}$ terminus of yeast Rad52, Genetics 161 (2002) 549-562.

[33] M. Lisby, R. Rothstein, U.H. Mortensen, Rad52 forms DNA repair and recombination centers during $S$ phase, Proc. Natl. Acad. Sci. U.S.A. 98 (2001) 8276-8282.
[34] M. Lisby, U.H. Mortensen, R. Rothstein, Colocalization of multiple DNA double-strand breaks at a single Rad52 repair centre, Nat. Cell. Biol. 5 (2003) 572-577.

[35] X. Zhao, E.G. Muller, R. Rothstein, A suppressor of two essential checkpoint genes identifies a novel protein that negatively affects dNTP pools, Mol. Cell 2 (1998) 329-340.

[36] C.S. Hoffman, F. Winston, A 10-min DNA preparation efficiently releases autonomous plasmids for transformation of Escherichia coli, Gene 57 (1987) 267-272.

[37] A.P. Davis, L.S. Symington, The yeast recombinational repair protein Rad59 interacts with Rad52 and stimulates single-strand annealing, Genetics 159 (2001) 515-525.

[38] G.R. Hicks, N.V. Raikhel, Protein import into the nucleus: an integrated view, Annu. Rev. Cell. Dev. Biol. 11 (1995) 155-188.

[39] D.A. Jans, C.K. Chan, S. Huebner, Signals mediating nuclear targeting and their regulation: application in drug delivery, Med. Res. Rev. 18 (1998) 189-223.

[40] J.A. Lloyd, D.A. McGrew, K.L. Knight, Identification of residues important for DNA binding in the full-length human Rad52 protein, J. Mol. Biol. 345 (2005) 239-249.

[41] N. Sugawara, G. Ira, J.E. Haber, DNA length dependence of the single-strand annealing pathway and the role of Saccharomyces cerevisiae RAD59 in double-strand break repair, Mol. Cell. Biol. 20 (2000) 5300-5309.

[42] F. Cortes-Ledesma, F. Malagon, A. Aguilera, A novel yeast mutation, rad52-L89F, causes a specific defect in Rad51-independent recombination that correlates with a reduced ability of Rad52-L89F to interact with Rad59, Genetics 168 (2004) 553-557. 